Dorin, A., "Artificial Life, Death and Epidemics in Evolutionary, Generative Electronic Art", Proceedings of 3rd European Workshop on Evolutionary Music and Art, Applications of Evolutionary Computing: EvoWorkshops 2005, Lausanne, Switzerland, March 30-April 1, 2005, Rothlauf et al (eds), Springer-Verlag Berlin, Heidelberg, pp448-457

\title{
Artificial Life, Death and Epidemics in Evolutionary, Generative Electronic Art
}

\author{
Alan Dorin \\ Centre for Electronic Media Art, Computer Science \& Software Engineering, \\ Monash University, Clayton, Australia 3800 \\ alandecsse.monash.edu.au
}

\begin{abstract}
This paper explores strategies for slowing the onset of convergence in an evolving population of agents. The strategies include the emergent maintenance of separate agent sub-populations and migration between them, and the introduction of virtual diseases that co-evolve parasitically within their hosts. The method looks to Artificial Life and epidemiology for its inspiration but its ultimate concerns are in studying epidemics as a process suitable for application to generative electronic art. The simulation is used to construct a prototype artwork for a fully interactive stereoscopic virtual-reality environment to be exhibited in a science museum.
\end{abstract}

\section{$1 \quad$ Motivation and Past Work}

Evolutionary, ecological simulations often converge with the population predominantly of similar genetic composition. For generative electronic art this may have undesirable aesthetic consequences, especially if the desired result is an exploration of diverse audio or visual solutions within the constraints of the work. For instance, it may be desirable that diverse visual and sonic forms be present in a simulated environment simultaneously and so the tendency of the population towards genetic impoverishment must be averted.

This paper focuses primarily on a notable omission from many Artificial Life models and publications, disease ${ }^{1}$. Typical ecological simulations model creatures competing for food, mating, fighting, and dieing. Yaeger's PolyWorld is a seminal example in which agents interact utilizing colour vision [1]. Todd has noted strategies for removing creatures from a population subject to a genetic algorithm but stops short of exploring different reasons for death in the population [2] (for example disease or suicide). Mascaro et al. have dealt specifically with suicide in a population of simple agents [3]. Ray's Tierra simulation eliminates elderly or ineffective population members with a "reaper". Also of interest is the emergence of "parasitic" code in his system [4].

The Artificial Life literature has much to say on co-evolution as a means of improving a genetic algorithm's performance through increased population diversity [5, 6]. This

\footnotetext{
${ }^{1}$ Little has been written in the Artificial Life literature on disease's counterpart decay either for that matter - a subject for future investigation.
} 
work is similarly inspired, only the simulations model agents in virtual worlds and do not optimize explicit fitness functions.

This paper borrows generally from ideas presented in all of the above literature, but its concerns are initially aesthetic and it specifically examines the process of epidemics - the transmission of disease through a population of susceptible individuals. Hence, most of the ideas for the research did not emerge from the publications above, but from ideas extracted from the literature of epidemiology and from art history. Both of these influences are surveyed below.

\subsection{A Brief Note on the Epidemic in Art}

It may seem a little crass or at best gothic to study the aesthetics of epidemics, yet for centuries plagues and disease have inspired artists to depict their horrors [7] and the fear of the ghastly death that often ensues [8]. Literary works such as The Masque of the Red Death (Poe 1842), the novel The Andromeda Strain (Crichton 1969), the film The Crazies (Romero 1973), and countless derivatives, maintain the cultural visibility and gory fascination of the epidemic. Contemporary art is no less concerned with epidemics. In particular, HIV/AIDS has driven many to artistic expression [9].

In the context of generative electronic art, the spread of infection is a biological process that may initially be treated without consideration of its emotional connotations. Such studies may lend themselves to a more thorough investigation of the potential of disease for application to software-based art. After the mechanisms of epidemics are understood this knowledge may be re-coupled with the emotional impact of the epidemic. The value of investigating biological processes and artificial life in the context of art are considered elsewhere [10].

\subsection{An Introduction to Models From Epidemiology}

A fully-cited history of the mathematical theory of epidemics is beyond the scope of this paper. The history leading to the classic model discussed below is provided in $[11,12]$.

At least since the 1920's, stochastic models of epidemics have been utilized. The standard model is based on a population of individuals who are either susceptible to a specific disease (susceptibles denoted $\mathbf{S}$ ) or infected with the disease and capable of transmitting it to others (infectives denoted $\mathbf{I}$ ). Population members who overcome a disease may become immune to further infection ${ }^{2}$ or may become susceptible once again depending on the particular disease. Population members who are immune to a disease or remain infected but through isolation cannot transmit it, are considered removed (denoted $\mathbf{R}$ ). The model as described is known as an SIR model. It may be modified slightly to provide fresh susceptibles through birth or immigration.

\footnotetext{
${ }^{2}$ Following a bout of a disease a victim may be deceased, alternatively their immune system may prevent repeat infiltration by the same virus.
} 
Some pertinent parameters of epidemic models are as follows. The period of time during which a disease exists entirely within an organism is known as the disease's latent period. The organism is not infective during this period. An incubation period often follows latency. During incubation the organism may not show outward sign of infection but is nevertheless infective. Usually once the incubation period is over, the victim of the disease is clearly marked by symptoms and can therefore be avoided by susceptibles.

Probabilistic epidemiological models that operate in discrete time steps are particularly suited to implementation in software. ${ }^{3}$ At any time step, the probability of a new case of the disease appearing is proportional to the number of susceptibles multiplied by the number of infectives. This basic model assumes random mixing of individuals in the population and does not allow for the complex interactions between physically separated sub-populations, nor for variable incubation or latent periods of a disease. Various extensions to allow for these phenomena have been added over the last fifty years. Some mathematical models and computer simulations deal with the spatial distribution of susceptibles along a line, across a lattice or over a network to overcome the inaccuracies due to the assumption of random mixing of the population. Cellular-automata and other discretized versions of the SIR method have been utilized also $[13,14]$. Some of these models have also incorporated disease carriers (e.g. some viruses are transferred by mosquito), and non-homogeneous populations.

The current threats of biological warfare and terrorism have raised the stakes in Western society for epidemiology. The U.S. National Institute of General Medical Sciences has devoted $\$ 1.6$ billion to a fledgling agent-based study of epidemics [15].

Like the U.S. project, this paper adopts agent modelling to represent the principles of epidemiology in an intuitive but realistic fashion. The motivation is, in this case, purely aesthetic. As shall be shown, the process of epidemic spread offers a means of varying the genetic and phenotypic diversity of a population and of capping its growth and density.

\subsection{Relevant Consequences of Basic Epidemic Theory}

There are two theories of epidemiology that are particularly relevant here. The first of these is known as the Threshold Theorem [16]: a disease cannot take hold in a population of susceptibles unless the population density is above a particular threshold. This value relates to the infectivity of a disease and the death and recovery rates it induces. If population density passes beyond the threshold, the disease will reduce the population to a level as far below the threshold as it was above it prior to the epidemic.

The Threshold Theorem has many consequences, one of which has come to be known as Herd Immunity [11, pp. 27-31]. This theory indicates that a calculable number less

\footnotetext{
${ }^{3}$ It is interesting to note that in the 1920's two American epidemiologists Reed and Frost demonstrated a discrete mechanical model in which coloured balls represented susceptibles and infectives.
} 
than the full population needs to be immunized to prevent an epidemic. Unfortunately the theory has been shown to provide inaccurate figures in practice, due to its assumption of random mixing in a population. Nevertheless, it highlights an important aspect of epidemics, namely that the spread of a disease is not dependent on the percentage of a population who are immune, but on the contact between susceptibles and infectives. When a population does not mix uniformly, the supply of susceptibles may be similarly irregular. ${ }^{4}$

\section{An Agent-Based Simulation of Infectious Disease Epidemics}

The present simulation runs in discrete time steps during which a population of agents (represented visually as coloured boxes of different sizes) moves freely about a continuous-space, toroidal world. The essential features of the model are described below, followed by a description of a prototype artwork that employs the new model.

\subsection{Agent Composition, Behaviour and Evolution}

Agents (coloured boxes) are able to detect the presence, colour and size of other boxes within their individual visual range. Boxes may move at their own speed towards or away from a box they see, or they may wander randomly. Their strategy for interacting with the world is based on a set of parameters that describe a path to travel based on the presence of a neighbour they "like", "dislike", or the absence of any visible neighbours. The like/dislike functions relate to each box's preferences for others of particular sizes and colours. The natural speed of movement of a box is inversely proportional to its volume. The box may speed up or slow down with respect to this value depending on how attractive or repulsive its goal appears.

Boxes meeting in space may find one another mutually attractive. If so, they may mate with one another and produce a single child per time step. The characteristics of the child are specified in terms of the genotype of each parent: an array of floatingpoint values coding an agent's dimensions and colour. The floating-point genotype also includes two colour templates, one that the agent seeks out in its companions (and potential mates) and the other from which it flees. A gene specifying a target partner size for the agent's likes and dislikes has also been implemented and operates in a similar manner.

The system behaves equally well with various schemes for crossover and mutation of the agent's genotype. This aspect of the system is not critical to its success but the method used employed a single crossover point and mutation of a gene in every child by a random amount between $+/-5 \%$.

\footnotetext{
${ }^{4}$ For example, if a socio-economic group is immunized against a disease, and these people do not mix randomly with people from other groups, an epidemic may still occur within the latter groups whilst the former is immunized. I.e. sub-group mixing is important in considering the spread of a disease.
} 
New births are subject to an overflow test of the available computer memory. If a birth would cause an overflow the request is refused. Following an unsuccessful request, a random member of the population may (or may not) be eliminated from the simulation to make room for future requests.

During each time step of the simulation, agents expend an amount of energy proportional to their volume to move and metabolise. Agent reproduction requires energy from each parent. This acts as a contribution to the initial energy of the offspring. Energy is gained by an agent each time step from the environment in an amount proportional to its upper surface area as if each box was equipped with a rooftop solar cell charging a battery. Boxes exhausting their energy supply are removed from the simulation (they die). Hence reproduction and movement both shorten the lifespan of a box if it is not able to glean sufficient energy to support itself.

Boxes age throughout a simulation. Those younger than a preset maturity age are unable to reproduce. Boxes that reach the end of their lifespan are removed from the simulation. These parameters are subject to evolution in the same way as the parameters discussed earlier.

\subsection{Disease Behaviour and Evolution}

The agents in the model are provided with features implementing infection and the transmission of diseases to others. Agents may visually detect the symptoms of a disease in others although this feature was not utilized in the current experiments.

A simulation disease may only occur within a host agent i.e. disease does not persist in the simulation environment. An agent is infected by a disease when it meets an infective and it is determined to be susceptible. A box of a particular colour is susceptible to a disease according to its match with the colour-signature of the disease. The closer the match between the two colours, the higher the probability the disease will be successfully transferred from the host to a susceptible in a time step during which the agents are in contact. The presence of an active disease in an agent blocks secondary infection.

Simulation diseases possess a devastation value. Highly devastating diseases remove large amounts of energy from their hosts every time step. Devastation is scaled by the match between a disease's colour-signature and the colour of the host. The closer the match, the more the devastation of the disease is scaled upwards. In addition, the higher the devastation and the closer the colour match to a susceptible, the more likely that agent will be infected.

A parameter determines the lifespan of a simulation disease in a particular host. The longer this is, the more energy a host needs to invest in total to overcome a disease. If a disease is overcome without the death of the host, the agent acquires immunity to the strain of the disease by adding it to an immunity list. Any further contact with this disease will result in an immune response that prevents the disease from infecting the agent a second time. 
A given instance of a disease is killed when its host is killed, irrespective of the lifespan of the disease. In addition to an individual lifespan, each disease has parameters indicating its dormant and incubation periods.

Real diseases such as viruses replicate and mutate within a host much more rapidly than the hosts themselves reproduce, circumventing the response of a host's autoimmune system. Consequently, it is possible for humans to repeatedly catch viruses such as the common cold and flu. In order to model this aspect of biology, within a specific host the simulation diseases undergo reproduction during every time step of their lifespan. This is modelled asexually and may mutate any aspect of the disease including its colour-signature, devastation, lifespan, incubation and latent periods. Mutation may also alter the parameter of each disease that determines how frequently it mutates during reproduction. Together all of these parameters allow the diseases to co-evolve with the more slowly evolving agent population.

The parameters for the disease and agents fully specify the features of the epidemic models discussed above. In conjunction with the agents' evolutionary model, a complex and flexible simulation has been devised that allows for studies of epidemics in non-homogeneous populations with non-random mixing. Fig. 1 illustrates the visualization scheme employed for the simulation.
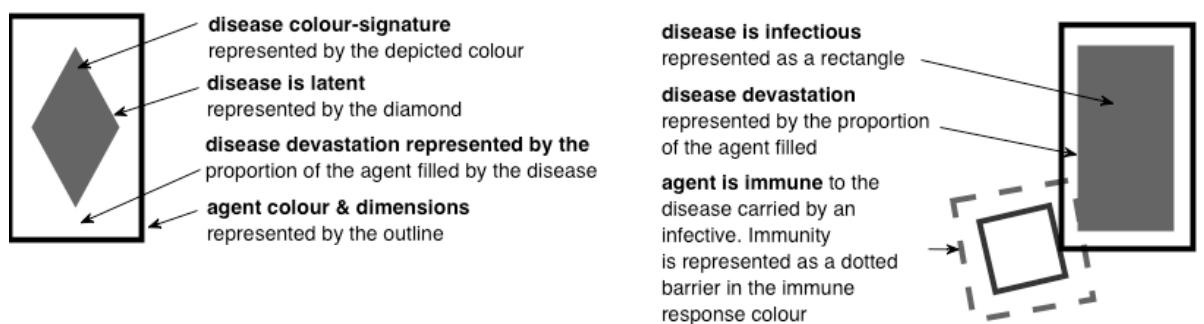

Fig. 1. The visualization scheme for agents and infection.

\section{$3 \quad$ Results}

It is beyond the scope of this paper to delve into the quantitative results of the simulation, this would be more fitting in the epidemiology literature. Instead, in keeping with the present aesthetic exploration, the results shall be described qualitatively.

As indicated in the motivation for this work, it had been noted that ecological simulations in which agents competed for resources (including mates and energy) often resulted in a genetically impoverished, homogeneous population. In the context of electronic media art, the resulting genetic drift through the phenotypic space was perceived by the artist to be quite beautiful, but nevertheless, perhaps a little boring. It was hoped that by introducing the element of disease to the population, diversity might be encouraged and uniformity exploited and eliminated by infection.

Happily, the disease did indeed exploit the population's uniformity when it arose. Disease also exploited populations of agents that clustered tightly together. In the 
absence of disease, boxes of particular colours and sizes often dominated a simulation, forming large colonies of potential mates. A typical screen shot after 14,000 time steps of the simulation without disease is reproduced in fig. 2(a). Fig. 2(b) illustrates a run after 14000 time steps with identical initial conditions, but in which the disease model was introduced. The diversity in dimensions, colour and spread of the population is far greater in fig. 2(b) than in fig. 2(a). In fact, after as few as 2500 time steps, the non-diseased model converges to homogeneity and does not break from this condition but drifts gently through genetic space. The population model incorporating disease seems to maintain its diversity indefinitely.
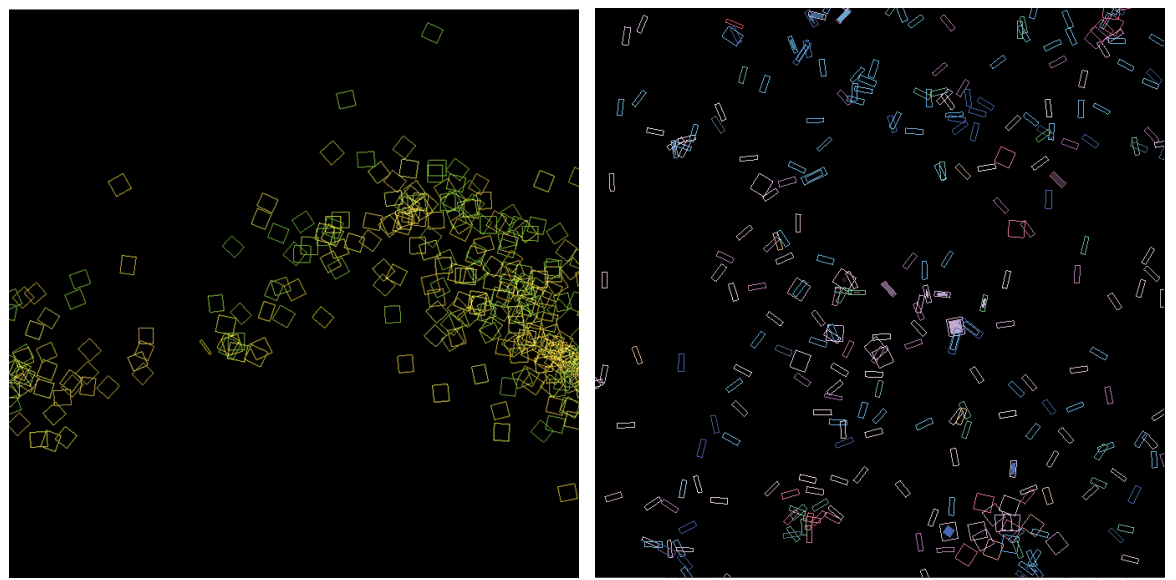

Fig. 2. Two simulation screenshots after 14,000 time steps:

(a) without the epidemiological model; (b) with the epidemiological model.

A disease simulation run involves the spontaneous appearance of a disease on average once every one-hundred-thousand agent updates. This new disease is generated with a colour-signature that matches the colour of the randomly selected agent. It is then infected with the disease and left to continue its travels. Apart from the coloursignature, all other disease parameters for the infection are randomly generated.

Depending on the parameters of the new disease, the traits of the infected agent and the population as a whole, the new disease may or may not cause an epidemic. The likelihood of an epidemic is specified by the Threshold and Herd Immunity theories described above. Some observed outcomes are described below along with the conditions giving rise to them in the present simulation environment.

Disease elimination (immediate). If the disease is insufficiently long-lived, or the population is insufficiently dense, or the host does not co-habit with others of a similar colour to itself, then the disease may fail to contact any susceptibles before it dies within the host. The disease will be eliminated from the population immediately.

Disease spread (immediate). A disease may mutate sufficiently within a host to infect susceptibles of a colour significantly different to the original host. If the host mixes amongst others of its kind they may become infected with the disease also. Occasionally the stochastic mechanism allows for a disease to infect a host coloured differently to its own signature. In this case, the devastation of the disease will be low 
in the infected host but the host nevertheless is able to infect other susceptibles. Such a host may be considered a "carrier" of the disease.

Disease elimination (eventual). If the disease manages to take a hold in the population it may nevertheless die out eventually if the number of susceptibles is reduced. This may happen when a sizeable proportion of the agents encountered by infectives is immune to the disease (even though the population as a whole may not have a significant number of immune members - see footnote 4 above). Circumstances like this arise when agents overcome the disease and acquire immunity, or when the disease is so devastating that it rapidly wipes out the supply of susceptibles before the agents are able to produce many offspring.

Disease spread (continual). A disease well-suited to its environment has sufficient lifespan to ensure it is passed from one susceptible agent to another. Such a disease also needs to be sufficiently devastating that it can be transferred successfully, but not so devastating that it kills off its supply of susceptibles. Diseases that fit these criteria also have to be sufficiently stable to avoid unwanted mutations that would render them ineffective, but sufficiently mutable so that they can keep infecting an evolving population of hosts. The simulation has given rise to diseases that meet all of these criteria and persist in the population for long periods of time.

Of particular interest are diseases that sustain themselves indefinitely when they are able to utilize susceptibles that are prolific breeders. Such diseases are able to spread through contact between mates who seek one another out (sexually transmitted diseases?) and also by contact between a parent and its newly born. Newly born agents may have traits slightly different to their parents so that occasionally one tends to wander off to seek its own preferred companions, taking the disease to infect others. As long as the disease remains latent for a sufficiently long interval, it will not kill or weaken the agent prior to its immigration to a further enclave.

\section{$4 \quad$ Artwork Prototype}

The eventual goal for this research is to establish an interactive, stereoscopic, virtual environment. The viewer's position will be monitored by a camera aimed in front of the viewing screen and mapped into a virtual location overlaid with the physical space. The overlay region is a section of the virtual world extending visually beyond the stereoscopic screen and therefore conceptually and apparently forming the interface between the virtual and real environments of the visitor's experience. ${ }^{5}$ See fig. 3 for a model and schematic of the system. The final work will have a more complex geometric model for the agents than the present wire-frame box.

By moving within the overlay region, visitors may attract the attention of the agents in the neighbourhood. Depending on the visual characteristics of the visitors, and the likes and dislikes of the agents, the virtual creatures may flee, approach, or remain

\footnotetext{
${ }^{5}$ Due to the nature of stereoscopic projection, the viewer will be unable to see agents behind or to the side of their own position but these will be simulated all the same. Agents in front of the viewer and apparently on either side of the screen will be visible.
} 
disinterested. It is anticipated that the traits of the audience that will be perceived by the agents include coarse approximations of an audience member's size, position and the presence of any colour as determined by processing a live video image of the overlay region for each screen of the visualization system. The eight-screen stereoscopic system for viewing the environment has been constructed and is currently situated in the Museum of Victoria in Melbourne, Australia. Software to monitor the video image has been partially written but has not yet been configured to operate with the simulation.

The prototype of the system above has been implemented employing virtual-human agents in place of the visitors. The apparent size of the virtual humans is normalized to match the dimensions of the box agents. Any colouration of the virtual agents (corresponding to any coloured clothing of the real humans on the video image) is treated as the presence of an infective disease in the human agent. Human agents do not die although they may leave the overlay region and thus the simulation. Box agents may mate with human agents if the box agents find the humans attractive. Offspring traits are determined by combining what is known about the human agent (based on their appearance on the video image) with the genes of the box agent.

If a "diseased" human agent encounters a susceptible box agent, the box may catch the disease and carry it into the virtual environment. Hence, the presence of human viewers wearing coloured clothes spreads disease that directly alters the evolution of the virtual population, culling populations of colour closest to the colours worn by the visitors.


Fig. 3. Artwork prototype: (a) installation visualization (agents will not be rendered as boxes in the final version); (b) schematic, overhead representation of a single screen. Humans (drawn as filled squares) and agents interact in the grey region "outside" the screen.

\section{Conclusions and Future Work}

A model of epidemics has been introduced to an evolutionary, agent-based simulation in order to increase the aesthetic interest of the population at any one time. The model improved the overall diversity of the population as desired and also encouraged its spread across the available virtual space. The behaviour of the epidemics modelled 
was in and of itself interesting to watch. A wide variety of disease outcomes emerged from the simulation, each an apparently plausible model of real-world outbreaks.

Much remains to be done. Besides the countless experiments that might be carried out with the epidemiological model for its own sake, the interactive artwork is still in its early stages. The video-processing software needs to be altered to work with the simulation (a time-consuming task) and more interesting visual forms need to be designed for the simulation agents. Nevertheless, even as it stands the simulation provides a fascinating experience for the informed viewer who understands the visualization system employed, and can interpret the coloured patterns appearing on the screen.

\section{References}

1. Yaeger, L.: Computational Genetics, Physiology, Metabolism, Neural Systems, Learning, Vision and Behavior or Polyworld: Life in a New Context. Artificial Life III, AddisonWesley (1992) 263-298

2. Todd, P.M.: Artificial Death. Jahresing 41 (German modern art yearbook) (1994) 90-107

3. Mascaro, S., Korb, K.B., Nicholson, A.E.: Suicide as an Evolutionary Stable Strategy. Advances In Artificial Life, 6th European Conference. Prague, Springer (2001) 120-133

4. Ray, T.S.: An Approach to the Synthesis of Life. Artificial Life II. Santa Fe, New Mexico, Addison Wesley (1990) 371-408

5. Cartlidge, J., Bullock, S.: Caring Versus Sharing: How to Maintain Engagement and Diversity in Coevolving Populations. 7th European Conference on Artificial Life. Dortmund, Germany, Springer-Verlag (2003) 299-308

6. Hillis, W.D.: Co-Evolving Parasites Improve Simulated Evolution as an Optimization Procedure. Artificial Life II. Santa Fe, New Mexico, Addison-Wesley (1990) 313-324

7. Boeckl, C.M.: Images of Plague and Pestilence : Iconography and Iconology. Truman State University Press Kirksville (2000)

8. Wolbert, K.: Memento Mori : Der Tod Als Thema Der Kunst Vom Mittelalter Bis Zur Gegenwart. Das Landesmuseum Darmstadt (1984)

9. Atkins, R., Sokolowski, T.W.: From Media to Metaphor : Art About Aids. Independent Curators Incorporated New York (1991)

10. Dorin, A.: The Virtual Ecosystem as Generative Electronic Art. 2nd European Workshop on Evolutionary Music and Art, Applications of Evolutionary Computing: EvoWorkshops 2004. Coimbra, Portugal, Springer-Verlag (2004) 467-476

11. Ackerman, E., Elvebeck, L.R., Fox, J.P.: Simulation of Infectious Disease Epidemics. Charles C Thomas Springfield, Illinois (1984)

12. Bailey, N.T.J.: The Mathematical Theory of Infectious Diseases and Its Applications. 2. Charles Griffin \& Company London (1975)

13. Willox, R., Grammaticos, B., Carstea, A.S., Ramani, A.: Epidemic Dynamics : DiscreteTime and Cellular Automaton Models. Physica A 328 (2003) 13-22

14. Martins, M.L., Ceotto, G., Alves, S.G., Bufon, C.C.B., Silva, J.M., Laranjeira, F.F.: A Cellular Automata Model for Citrus Variagated Chlorosis. eprint arXiv : cond-mat/0008203 (2000) 10

15. Pilot Projects for Models of Infectious Disease Agent Study (Midas). Webpage. National Institute of General Medical Sciences (NIGMS) http://grants.nih.gov/grants/guide/rfafiles/RFA-GM-03-008.html (Accessed: October 2004)

16. Kermack, W.O., McKendrick, A.G.: Contributions to the Mathematical Theory of Epidemics. Proceedings of the Royal Society, London 115 (1927) 700-721 
